\title{
Tactile roughness of grooved surfaces: A model and the effect of friction
}

\author{
M. M. TAYLOR and S. J. LEDERMAN \\ Defence and Civil Institute of Environmental Medicine \\ 1133 Sheppard Avenue West, P.O. Box 2000, Downsview, Ontario, Canada M3M 3B9
}

\begin{abstract}
Previous experimental data on the roughness of grooved aluminum tiles provide a data base against which to test theories of roughness perception. A model based on the static deformation of the skin touching the stimulus tile is developed, and 11 parameters of the deformation are individually compared with the experimental data. All parameters were tested first in an approximate way, and then the better parameters were recalculated in a more exact manner. Three parameters, the depth to which the finger penetrates the groove, the cross-sectional area of the finger within the groove, and the cross-sectional area of the deviation of the skin from its resting position, all predict the roughness well as a function of finger force and groove width. The last of the three predicts roughness best as a function of land width, and is tentatively preferred as "the stimulus for roughness." All predictions from the static model indicate that variation of the coefficient of friction between skin and tile should have little or no effect. This counterintuitive prediction was confirmed by an experiment.
\end{abstract}

Although psychophysics has been with us for over a century, there has been little discussion and no agreement on what constitutes the stimulus for roughness. There are two probable reasons for this lack: stimuli graded in an appropriate physical dimension are hard to manufacture, and knowledge of the tactile receptor systems and their functions is quite limited. There is so little agreement on the stimulus for roughness that the present authors were able to engage in a difference of opinion with their co-author in a recent chapter on texture perception (Taylor, Lederman, \& Gibson, 1973). Gibson claimed that the perceived roughness of a surface depended on the frequency of vibration induced by the passage of the finger over the surface, while Taylor and Lederman held that vibratory energy, rather than its frequency, was responsible. The present paper introduces the hypothesis that neither vibratory frequency nor vibratory energy is the stimulus for perceived roughness; the deformation of the skin rather than the energy involved in the deformation is the important factor.

A recent series of experiments (Lederman, 1973, 1974; Lederman \& Taylor, 1972) on the perceived roughness of grooved aluminum tiles (or plates) has provided a data base against which theories of roughness may be tested. The experiments have shown that the perceived roughness of the plates depends strongly on the width of the grooves, less strongly on the applied finger force, slightly on the width of the undisturbed surfaces ("lands") between the grooves, and hardly at all on the speed with which

This is DCIEM Research Paper No. 73-RP-981. S. J. Lederman is now at Queen's University, Kingston, Canada. the finger moves over the tile. There are small subtle interactions among these variables, but they are unimportant compared to the major effects. Some of the results, especially at the lower roughness levels, may be due to artifacts of stimulus production (Lederman, 1974). A theory of perceived roughness should ideally account for all the effects and interactions, and should explain how the differences in stimulus production methods affect the perception of the smoother plates.

In Part 1 of this paper, a model for perceived roughnes's is presented which accounts for the major features of the data and which, by extension, can be shown to be compatible with the more subtle interactions. Contrary to intuition and to data in the literature, the model predicts that the coefficient of friction between skin and plate should have very little effect on perceived roughness. Part II of the paper presents an experiment in which the friction coefficient was varied without changing the geometry of the plates. The results were in agreement with the prediction from the model.

\section{PART I: \\ ANALYSIS OF THE TOUCHING PROCESS}

\section{Foundations of the Model}

The model rests on several presuppositions, which in some cases have strong supporting evidence, but in others must be taken as pure assumption. We consider these presuppositions first.

The perception of roughness must be due to the deformation of the skin. Since simply squashing the skin, or passing it over a rippled surface such as bubbled glass, does not give rise to a sensation of 
roughness, it is clear that the deformation must have a scale small compared to the width of the finger. This is more of a statement of fact than an assumption, but is required in the development of the model.

A second commonplace fact about roughness is that it can be perceived only when the surface moves relative to the skin. As Katz (1925) mentioned, static irregularities are very quickly adapted out. Despite this fact, both Katz (1925) and Lederman (1974) found that changing the speed of the hand over the surface had very little effect on perceived roughness, except that smooth objects felt slightly smoother at high speed (10 in. $/ \mathrm{sec}=25 \mathrm{~cm} / \mathrm{sec})$. The small effect of hand speed suggests either that there is a strong constancy effect operating or that the stimulus for roughness does not depend on vibration frequency. Vibration frequency is taken to be the frequency with which bumps pass under a particular point on the skin. A parsimonious position, and the one which gives rise to the model presented here, is that the only restriction on hand speed is that the skin deformation should change rapidly enough to prevent adaptation and that the hand should move slowly enough that skin deformation can occur. Within these limits, the actual hand motion is irrelevant.

The irrelevance of hand speed leads to the assumption that the actual movements of the skin in assuming its various deformations are also irrelevant. What matters is the shape of the skin at any moment. Accordingly, the present model uses a static analysis of the touching process, in which the position of the skin of an idealized finger pressing on a groove is determined.

Von Gierke, Oestreicher, Franke, Parrack, and von Wittern (1952) and Moore and Mundie (1972) have studied the bulk impedance of tissue excited by a piston. While most of the results are not directly relevant to the present problem, one of the observations of von Gierke et al. (1952) bears directly on the assumptions underlying the model. This is the fact that, on both the upper arm and the thigh, the speed of surface waves was about $160 \mathrm{~cm} / \mathrm{sec}$. It is unlikely that the speed of surface waves on the fingertip is less than on the softer tissues of the thigh and upper arm. Since the most rapid finger movement used in any of the experiments whose data is to be fitted by the model was about $25 \mathrm{~cm} / \mathrm{sec}$, disturbances of the skin surface could propagate much faster than the finger moved over the plate. This is a necessary condition for a quasistatic model to be valid, and is the primary condition required for the validity of the particular model proposed here. Since the speed of the surface waves is so high, the skin has plenty of time to assume an average position like that predicted by the quasistatic model.

In order to make numerical predictions, both the finger and the grooves of the aluminum plates must be idealized. The finger is represented by a membrane enclosing a fluid. The membrane is infinitely flexible, but has a finite coefficient of elasticity. The fluid assures that if there is pressure within the finger, it is exerted normally to the skin at all points. While the real tissue is both viscous and compressible, the only property required for the model is that the pressure be equalized and normal to the surface. The high speed of the surface waves found by von Gierke et al. (1952) assures that the assumption is at least approximately true, provided that the viscous damping is adequate. It may seem strange at first sight to assume infinite flexibility for the skin, and the assumption is not true in fact. But our own skin and that of our subjects is quite flexible enough to take a good impression of even the smaller grooves used in the experiment. Hence, it must be considered flexible on the scale considered here. We know of no formal determinations of skin flexibility either in vivo or in vitro. The stretching of the skin is assumed to be proportional to the tension within the skin. Real skin does not stretch linearly (Tregear, 1966; Yamada, 1970), but if a small initial tension is applied, subsequent stretching is nearly proportional to further increments of tension. We assume that blood pressure within the fingertip provides this small initial tension, and that the skin stretches proportionately to tensions induced by touching surface irregularities.

For the purposes of the analysis, the grooves in the tiles are assumed to be perfectly rectangular and to occur at exactly equal intervals. The real tiles showed small irregularities near the groove edges (Lederman, 1974) which may have been responsible for the apparent anomalies of the data for very smooth tiles. Such irregularities are ignored in the analysis, although we have more to say about them later.

\section{Analysis}

Following the assumption that skin deformation is responsible in some way for the experience of roughness, the analysis proceeds by determining what shape the skin assumes under the influences of the various forces at play during the touching process. Since we consider only a quasistatic model, dynamic forces are ignored and the only forces acting on the skin are pressure from the fluid within, reaction forces from the land surfaces of the plate, and tension in the skin. The latter is always within the surface of the skin, the two former always perpendicular to the skin.

The various forces and geometrical variables considered are shown in Figure 1, which depicts four of the grooves in a tile on which the finger is pressing. The grooves, as in all experimental conditions, are narrow compared to the width of the finger. The first (leftmost) groove shows the forces involved. The total finger force, $F$, creates a pressure, $P$, within the finger. A part of the force, $f_{g}$, is exerted on the skin 


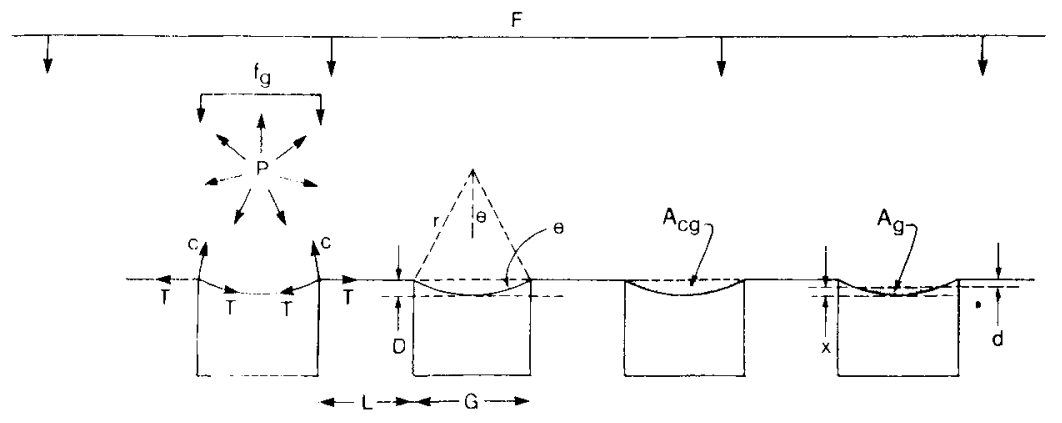

Figure 1. The geometry of the idealized finger pressed onto a series of grooves. Different parameters of the deformation are shown in each of the four grooves. The finger assumes the form of a circular arc within each groove under the influence of pressure induced by the force of touching. For details of the parameters, see text. directly over each groove, forcing the skin down into the groove. Since the force is at all points perpendicular to the skin, the skin must assume an arc of a circle, becoming stretched in the process and creating a tension, $T$, in itself. The tension, $T$, produces a reaction force, $\mathrm{C}$, at the groove corner, and the vertical components of the two reaction forces at the two corners exactly balance $f_{g}$. The portion of the finger force applied over the land is immediately balanced by a reaction force from the land, and is of interest only insofar as it and the coefficient of friction control the amount of skin that can slip off the land into the groove.

The second groove in Figure 1 indicates that the skin has been depressed to a maximum depth, D, in the groove of width $G$, and that the arc formed by the skin has a radius, $r$, and meets the groove edge at an angle, $\Theta$, from the horizontal (alternatively, the angle subtended by the arc is $2 \theta$ ). The total cross-sectional area of the depression is $A_{c g}$ (shown in the third groove). The fourth groove shows another interesting parameter, $\mathrm{A}_{\mathrm{g}}$, the cross-sectional area of the amount by which the skin is depressed below its overall mean level. If it is considered that the land raises the skin by an amount $\mathrm{d}$ and, in compensation, the pressure forced it down into the groove an amount $x$, then the cross-sectional area of the raised portion is by definition equal to $A_{g}$, the cross-sectional area of the depressed portion. To anticipate the final result, $\mathrm{Ag}_{\mathrm{g}}$ summed over all grooves in which the finger rests appears at present to be the most promising candidate for the stimulus for roughness. When generalizing to random surfaces, of course, all parameters called "areas" here must be replaced by the corresponding volumes.

Most of the letters in Figure 1 refer to parameters which could conceivably be detected by appropriate receptors and coded into perceived roughness. There are other possibilities not depicted in Figure 1. In all, we have seriously considered 11 candidates. In no particular order, these are: (1) $G$, unsupported skin span, which could conceivably serve as an index of the scale of surface irregularities; (2) $\mathrm{T}$, the maximum tension in the skin; (3) $\Delta S_{g}$, the amount by which the skin stretches when it is depressed into a groove;
(4) $\Delta S$, the total amount by which the skin stretches summed over the whole finger width; (5) $\Theta$, which represents the maximum flexion of the skin at any point; (6) D, the maximum depth difference between points on the skin; (7) $\mathrm{C}$, the maximum force applied at any point on the skin; (8) $A_{c g}$; (9) $A_{c}$, which is $A_{c g}$ summed over the width of the finger; (10) $A_{g}$; and (11) A, which is $A_{g}$ summed over the width of the finger. Doubtless, we have overlooked other possible candidates, even within the restrictions of the static model.

\section{Gross Analysis}

The data of the experiments (Lederman, 1973, 1974; Lederman \& Taylor, 1972) suggest the crude generalization that perceived roughness is a power function of groove width and of applied finger force, provided that the tiles are not too smooth. Within the power-function range, the exponent for groove width is approximately four times the exponent for finger force. Accordingly, we can say as an approximation that roughness is a function of $\mathrm{FG}^{4}$. Any candidate that is roughly a function of $F G^{4}$ is worth close study. It should be noted that $F^{1 / 2} G^{2}$ is a function of $F G^{4}$, but $F^{2} G^{4}$ is not. We do not require that the candidate parameters bear the same functional relationship to $\mathrm{FG}^{4}$ as does roughness, since perception is only a function of stimulation, rather than being equal to the stimulation.

Each of the candidate parameters may be calculated in terms of an expression in $\Theta$. For all except $A_{g}$ and $A$, a small-angle approximation to $\sin \Theta(=\Theta)$ and to $1-\cos \Theta\left(=1 / 2 \Theta^{2}\right)$ reduces the expression to a power function for the candidate in terms of $F$ and $G$. The exponents of $F$ and $G$ in these expressions, together with their ratios, are presented in Table 1 for two limiting values (zero and infinity) of the friction coefficient. Those candidates whose ratios do not come close to 4 may be immediately discarded from more exact consideration. In discarding candidates, due allowance must be made for the crudity of the small-angle approximations. Nevertheless, $G, T, \Delta S_{g}, \Delta S, \Theta, C$, and $A_{c g}$ may be discarded at this stage, leaving only $\mathrm{D}, \mathrm{A}_{\mathrm{C}}$, and the untested $\mathrm{A}$ and $\mathrm{Ag}$ for more detailed analysis. 
Table 1

Exponents of $F$ and $G$ for Various Candidate Parameters, Assuming Either Infinite Friction or No Friction

\begin{tabular}{|c|c|c|c|c|c|c|}
\hline \multirow[b]{2}{*}{ Parameter } & \multicolumn{3}{|c|}{ No Friction } & \multicolumn{3}{|c|}{ Infinite Friction } \\
\hline & $\begin{array}{l}\text { Exp } \\
\text { of } F\end{array}$ & $\begin{array}{r}\text { nents } \\
\text { of } G\end{array}$ & Ratio & $\begin{array}{l}\text { Exp } \\
\text { of } F\end{array}$ & $\begin{array}{r}\text { nents } \\
\text { of } G\end{array}$ & Ratio \\
\hline G & 0 & 1 & - & 0 & 1 & - \\
\hline $\mathrm{T}$ & $3 / 4$ & $3 / 4$ & 1 & $2 / 3$ & $2 / 3$ & 1 \\
\hline$\Delta \mathrm{S}_{\mathrm{g}}$ & $3 / 4$ & $7 / 4$ & 2.3 & $2 / 3$ & $5 / 3$ & 2.5 \\
\hline$\Delta S(G>>L)$ & $3 / 4$ & $3 / 4$ & 1 & $2 / 3$ & $2 / 3$ & 1 \\
\hline$\Delta \mathrm{S}(\mathrm{G}<<\mathrm{L})$ & $3 / 4$ & $7 / 4$ & 2.3 & $2 / 3$ & $5 / 3$ & 2.5 \\
\hline$\theta$ & $1 / 4$ & $1 / 4$ & 1 & $1 / 3$ & $1 / 3$ & 1 \\
\hline $\mathrm{D}^{*}$ & $1 / 4$ & $5 / 4$ & 5 & $1 / 3$ & $4 / 3$ & 4 \\
\hline $\mathrm{C}$ & 1 & 1 & 1 & 1 & 1 & 1 \\
\hline$A_{c g}$ & $1 / 4$ & $9 / 4$ & 9 & $1 / 3$ & $7 / 3$ & 7 \\
\hline$A_{c}(G>>L)^{*}$ & $1 / 4$ & $5 / 4$ & 5 & $1 / 3$ & $4 / 3$ & 4 \\
\hline$A_{c}(G<<L)$ & $1 / 4$ & $9 / 4$ & 9 & $1 / 3$ & $7 / 3$ & 7 \\
\hline
\end{tabular}

*Parameter retained for more exact analysis.

\section{Further Analysis of the Ideal Model}

Whereas the power function approximations could be obtained with no great difficulty, a more precise analysis required numerical calculation and the expenditure of appreciable computer time. Accordingly, only those candidates with appropriate power ratios were included in the later analysis.

Several items had been omitted in the approximate analysis. For example, no account was taken of the fact that the fingertipe spreads out with increasing finger force. For the numerical analysis, this spread was measured, using five subjects, two male and three female. A tile was put in the touch apparatus (Lederman \& Taylor, 1972), with a piece of paper on top. The subject covered his middle finger with fingerprinting ink and held it in place over the tile in the attitude of touching. The plate was held by the second and fourth finger and then released, to be stopped by the inked middle finger. The applied force was controlled by the balance counterweights, as it was in the touching experiments. Six clear fingerprints were made at each of four forces $(1,4,9$, and $16 \mathrm{oz} ; 1 \mathrm{oz}=28 \mathrm{~g}$ approximately). Fingerprints proved to be nearly elliptical in shape, although the size varied for the different subjects. The effect of changing force was the same for all subjects, pad area being approximately proportional to the 0.15 power of the force. In the numerical analyses, the geometric means of the pad areas actually observed were used, rather than this power-law approximation.

Numerical values of the deformation parameters, not functional relationships, were determined in the numerical analyses. To obtain these actual values, estimates for the skin coefficient of elasticity, $k$, and the skin-tile coefficient of friction, $\mu$, were needed, since both appear in the expressions for the candidate parameters. Unfortunately, neither was readily available in the literature.

To derive a reasonable figure for the coefficient of elasticity of the skin in the linear region of the stress-strain curve, we took a curve presented by Yamada and Evans (1970, Figure 199), assumed a fingertip skin thickness of $0.5 \mathrm{~mm}$, and derived a coefficient of $0.08 \mathrm{~mm} \mathrm{~g}^{-1}$. The Yamada and Evans graph is the most relevant we could find, but it is rather small and deals with skin on other parts of the body. Accordingly, we have used this figure of $0.08 \mathrm{~mm} \mathrm{~g}^{-1}$ only as a guide, and have made the numerical calculations using estimates within an order of magnitude on either side. While the numerical values of the candidate parameters are strongly affected by changes in the assumed value of $k$, the functional relationships with $F$ and $G$ are altered only slightly. The real value of $k$ must lie somewhere in the range of the values tested, and we believe that the calculations are valid in this respect.

The coefficient of friction has less effect on the candidate parameters than does the coefficient of elasticity. After completion of the analytic studies on the model, it proved desirable to measure the coefficient of friction directly (see Part II of this paper), but for the analysis, a most likely value of 0.5 was assumed. Values from zero to $10^{4}$ were used in various analyses, but the range 0.1 to 2.0 was studied most thoroughly. Changing the coefficient from zero to 2.0 has a barely discernible effect on the calculated values, and increasing the coefficient to $10^{4}$ only decreases the parameter values slightly for the wider land tiles (Lederman, 1973, Figure V-1).

\section{Calculations}

The method of calculation was similar for each of the candidate parameters. Details for all of them are given by Lederman (1973), and two examples are shown in Appendix 1. Briefly, the technique is based on the mathematical fact that both the dimensionless quantity $\mathrm{kf}_{\mathrm{g}}$ and the candidate parameter could be expressed as a function of $\Theta$. The question of interest was what value was taken by the candidate for a given value of the finger force, $F$, and groove width, G, which are readily translated into $\mathbf{k f}_{\mathrm{g}}$ if a value of $\mathrm{k}$ is assumed. The value of $\Theta$ corresponding to the given value of $\mathrm{kf}_{\mathrm{g}}$ was determined by an iterative process, and the value of the candidate was found directly from this value of $\Theta$. The whole procedure was done for many combinations of assumed values of $k$ and of $\mu$, over the whole range of tile geometries and forces used in the experiments.

Initial results showed that of the four remaining candidates, $\mathrm{Ag}$ could be discarded as a candidate, leaving $D, A_{c}$, and $A$ for full analysis. Selected values for these remaining candidates are shown in Figures 2.4 for three sets of tiles, corresponding to those of Lederman's (1974) Experiments IV and III, in that order. These sets are a set (Experiment IV) with land width $0.010 \mathrm{in} .(0.25 \mathrm{~mm})$ and groove width 
Figure 2. Predicted values of $D$, the depth of penetration of finger into groove, as a function of finger force and groove width. Assumed parameters: $\mathrm{k}=0.08 \mathrm{~g} \mathrm{~mm}^{-1}, \mu=$ 0.5.

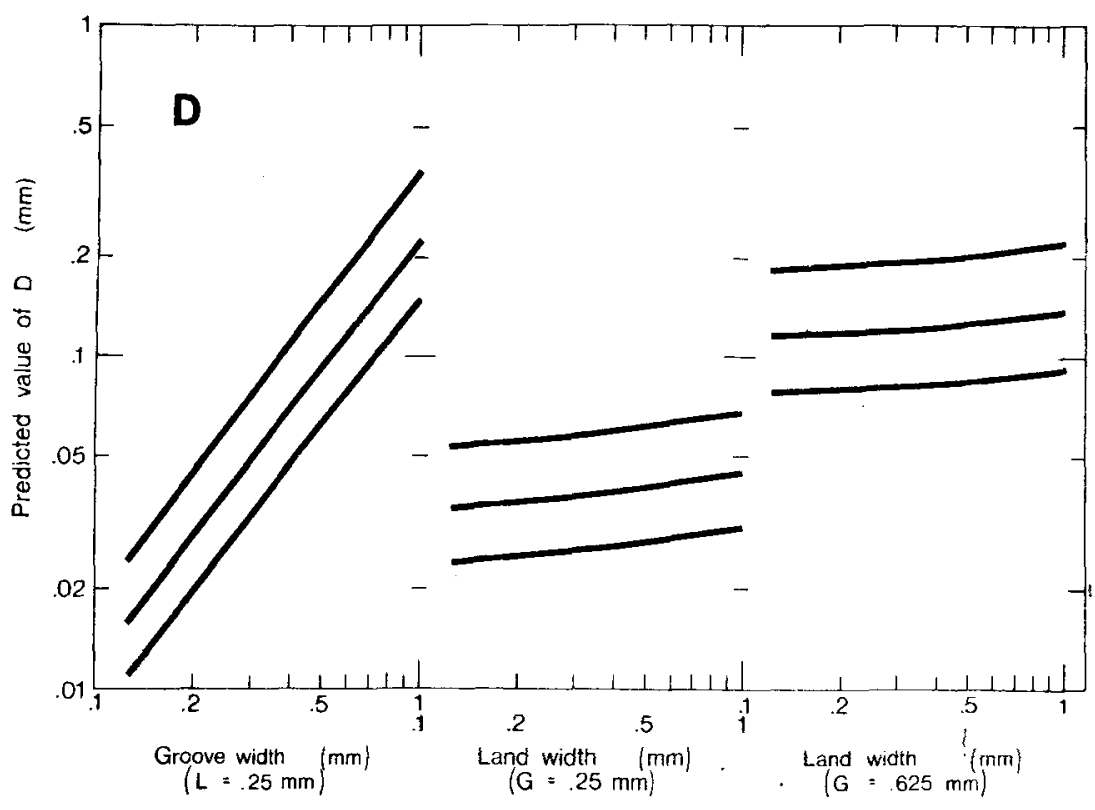

varying from 0.005 to 0.040 in. $(0.125$ to $1.0 \mathrm{~mm})$ and a set (Experiment III) with groove width 0.010 in. $(0.25 \mathrm{~mm})$ and land width varying from $0.007 \mathrm{in}$. $(0.18 \mathrm{~mm})$ to $0.040 \mathrm{in}$. $(1.0 \mathrm{~mm})$, and a similar land-varying set (also Experiment III) with groove width fixed at $0.025 \mathrm{in} .(0.625 \mathrm{~mm})$. In other words, there was one groove-varying and two land-varying sets of tiles. Figure 5 shows the corresponding experimental values for perceived roughness. Experimental perceived roughness is plotted against predicted parameter values for the three candidates in Figures 6-8. In Figures 6-8, the perceived roughness values from Experiment IV have been augmented by $0.227 \log$ units. The numerical scales used by the subjects in the two experiments were not necessarily the same, and this adjustment brings them into alignment. The solid lines in Figures 6-8 represent a slope of unity, and are not fitted to the data.

Surprisingly, even in the exact numerical analysis with trigonometric formulae, all the candidates give results that closely approximate power functions as groove width varies. All give approximately the correct relationship between the effects of finger force and of groove width. All fail to predict the fact that the perceived roughness of tiles with narrow grooves is effectively independent of groove width or finger force (the "leveling" effect). Discrimination among the candidates must depend on their prediction of
Figure 3. Predicted values of $A_{c}$, the total cross-sectional area of finger penetration into grooves, summed over the whole area of the fingertip in contact with the tile. Assumed parameters as in Figure 2.

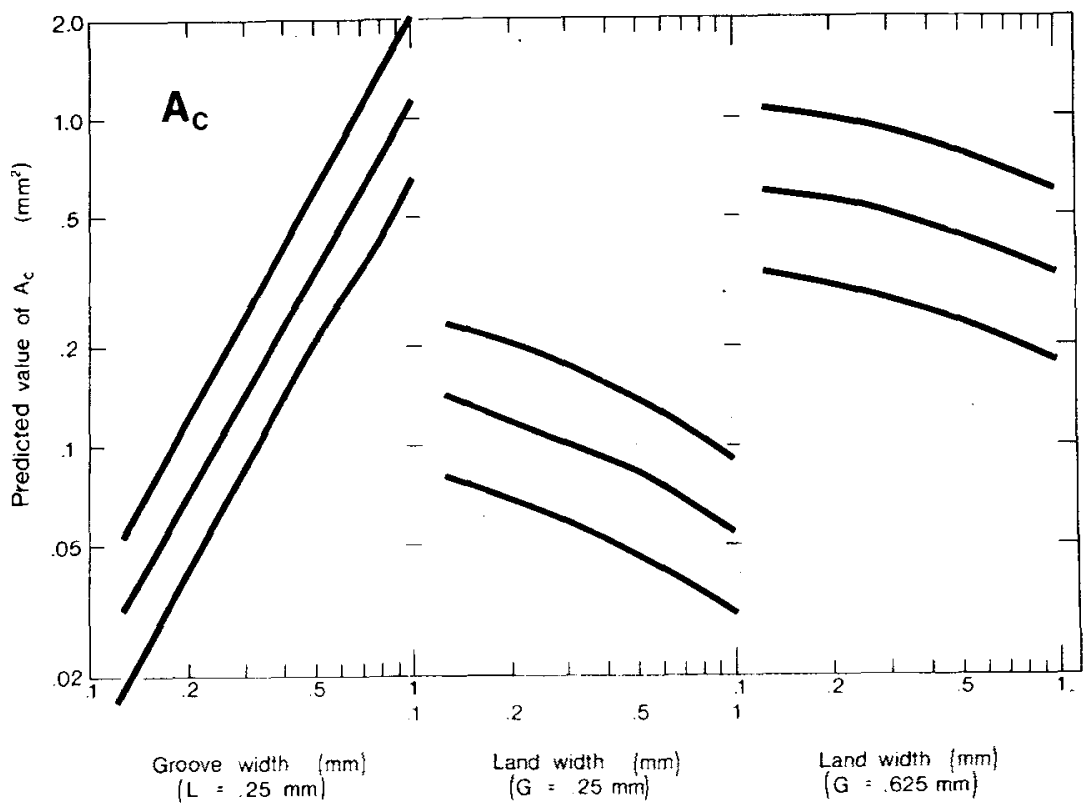


Figure 4. Predicted values of $A$, the cross-sectional area of the deviation of the skin from its resting position, summed over the whole area of the fingertip in contact with the tile. Assumed parameters as in Figure 2.

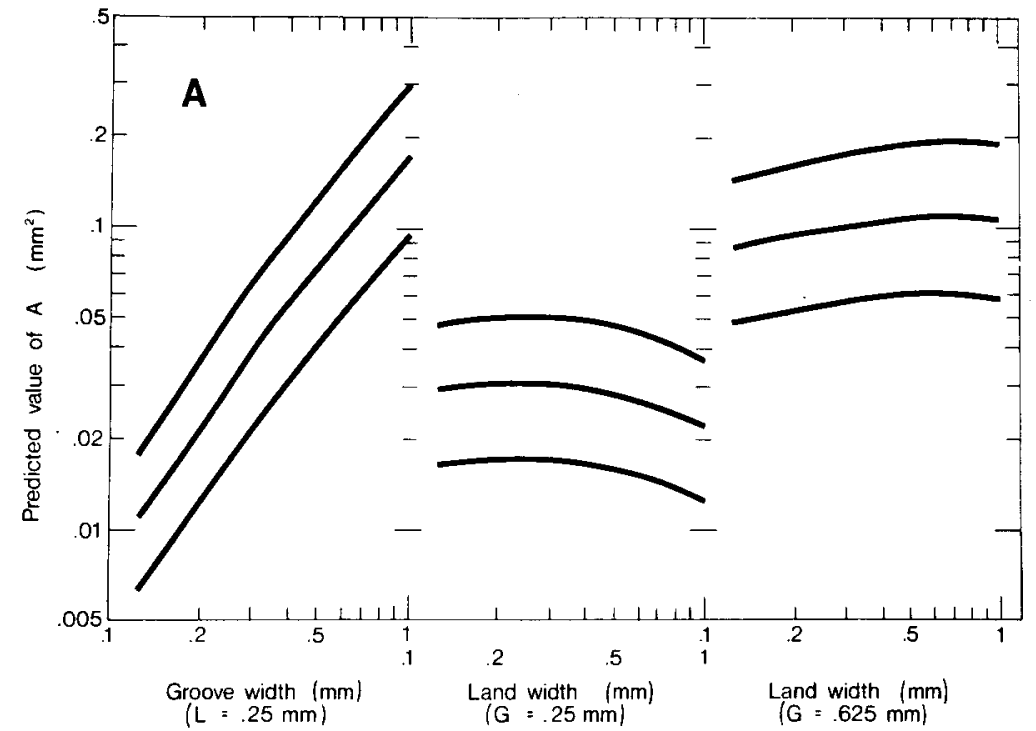

$\begin{array}{ccc}\text { Groove width }(\mathrm{mm}) & \text { Land width }(\mathrm{mm}) & \text { Land width }(\mathrm{mm}) \\ (\mathrm{L}=.25 \mathrm{~mm}) & (\mathrm{G}=.25 \mathrm{~mm}) & (\mathrm{G}=.625 \mathrm{~mm})\end{array}$ roughness as land width varies; experimentally, force has little effect and roughness decreases with increasing land width when the grooves are narrow; when the grooves are wider, force has its ordinary effect and land width has none. All candidates predict force to have its normal effect regardless of groove width; this failure is another manifestation of the unpredicted leveling effect. As Lederman (1974) has argued, the leveling effect is probably a stimulus production artifact which cuases the tiles to differ from the ideal form assumed in the analyses. Hence we may cautiously disregard the failure of the candidates to predict it. Later, we show that A, at least, may respond in the correct way to the true forms of the tiles as revealed by scanning electron microscopy. For the present, however, we shall ignore the leveling effect.

The parameter $\mathrm{D}$ increases with increasing land width, for both narrow and wide grooves. This is contrary to the experimental results, and reduces the credibility of $D$ as the stimulus for roughness. $A_{\mathcal{C}}$, the total cross-sectional area of depression referred to the level of the lands, declines with increasing land width for both wide and narrow grooves, though less for wide than for narrow grooves. The amount of the decline is too large in the case of the narrow grooves, and no decline should be evident with the wide grooves, if the experimental data are to be believed. $\mathrm{A}$, the total cross-sectional area referred to the mean level of the skin, behaves more or less correctly,
Figure 5. Perceived roughness as a function of finger force and groove and land width. Data from Lederman (1974; Experiments III and IV).

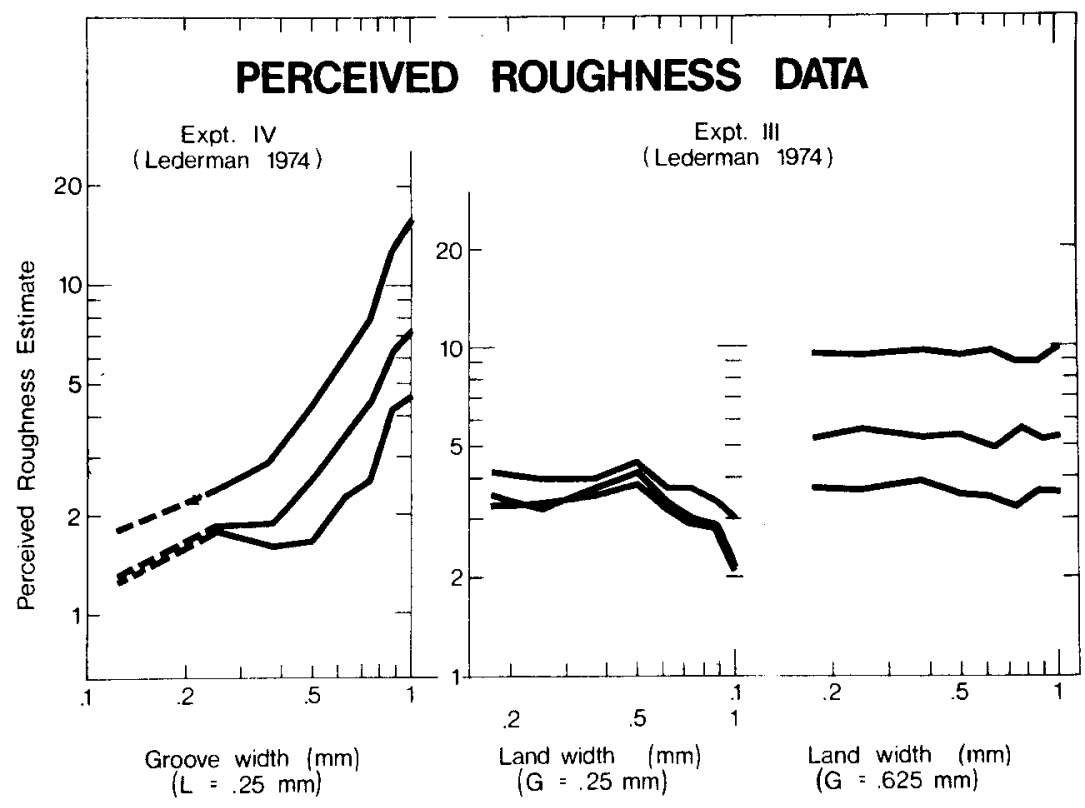


Figure 6. Perceived roughness plotted against predicted values of the parameter $D$ (maximum depth of the finger penetration into the groove) for all experimental conditions of Lederman (1974; Experiments III and IV). The perceived roughness data for Experiment IV have all been increased by 0.227 log units, to make their absolute level compatible with the absolute level of the perceived roughness data from Experiment II. The solid line has a slope of unity, and is not fitted to the data.

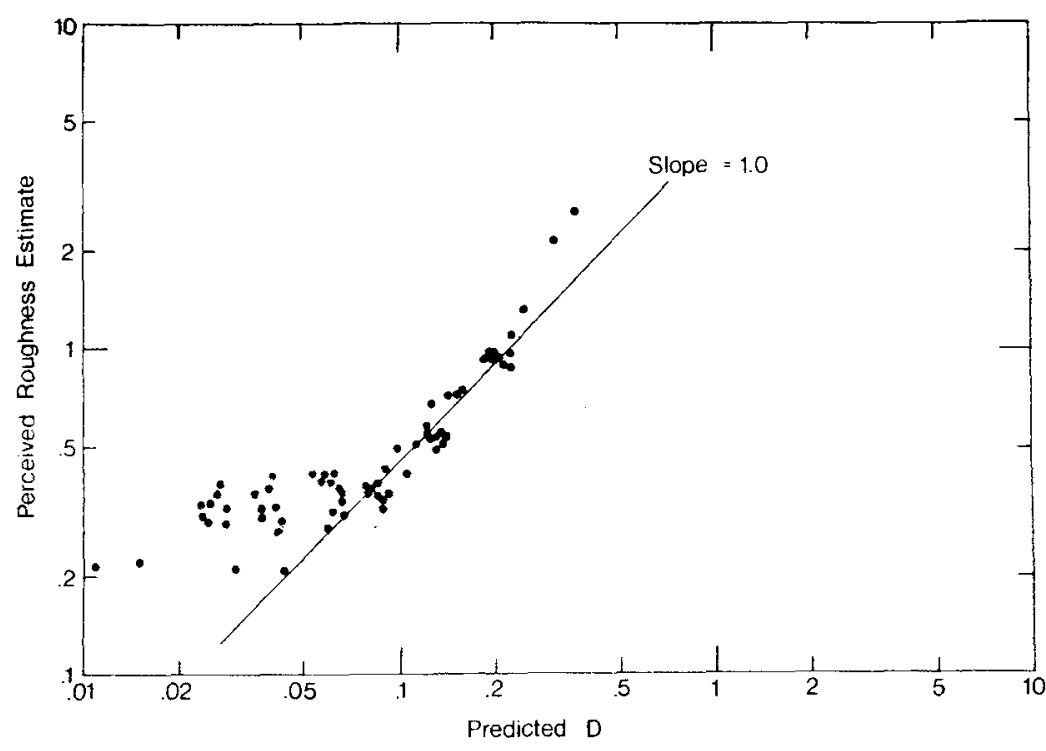

declining slightly, especially for wider lands, when the grooves are narrow, and remaining constant but with a slight upward convexity when the grooves are wider. Apart from the failure to predict the leveling effect, A behaves very like the perceived roughness as a function of force, groove width, and land width.

The scatter graphs of Figures 6-8 do not discriminate among the three candidates, but they do serve to show that prediction failure occurs only because the perceived roughness does not become very low when the predictions indicate that it should.

\section{Production Artifacts}

We must now consider the potential effects of stimulus production artifacts. The ideal grooves are rectangular cuts in a flat plate. The real grooves produced by machining followed by burnishing
(S-plates) have somewhat torn, sloped edges with a raised burr at the edge of the land surface (Lederman, 1974). The burr is smoothed by the burnishing, and the pair of burrs at the two sides of the land look in the scanning electron microscope pictures rather like wavy railway tracks. The effect is that there are really two grooves, one "official" and a very shallow "unofficial" one between the "railway tracks" on what is supposed to be the land.

The grooves produced by electric discharge machining (EDM-plates) also differ from the ideal form. These tiles were not burnished after the grooves were produced because their nominal tolerances were much better and because there were no obvious burrs. Nevertheless, the tiles had a consistent "matte" look and feel, as opposed to the smooth look of the S-plates. In the scanning electron microscope
Figure 7. Perceived roughness plotted against predicted values of the parameter $A_{c}$ (cross-sectional area of finger penetration into grooves). Conditions as for Figure 6.

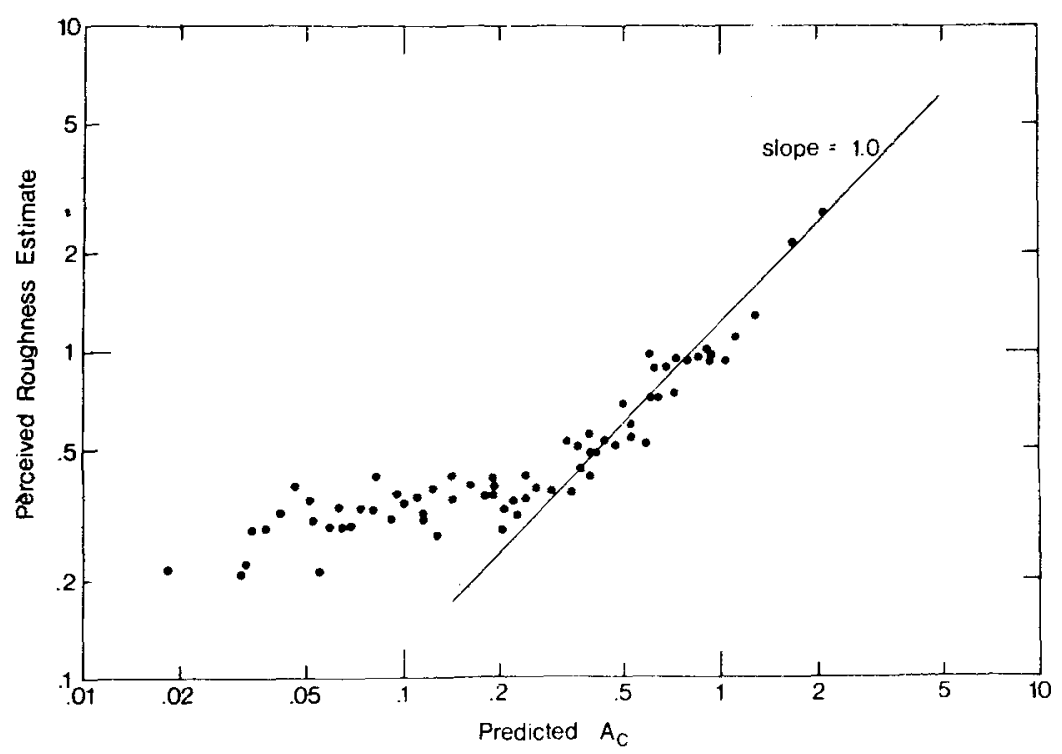


Figure 8. Perceived roughness plotted against predicted value of the parameter $A$ (total cross-sectional area of the deviation of the skin from its resting configuration). Conditions as in Figure 6.

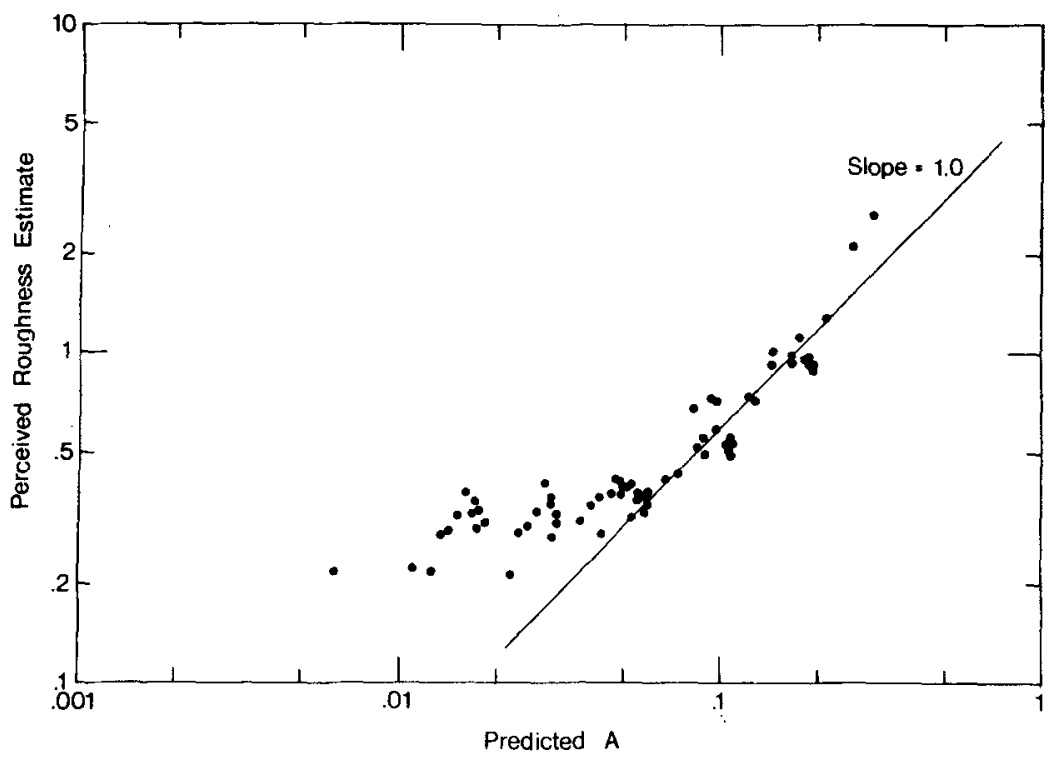

pictures, the grooves are rectangular in cross section, but at the edges of the lands are burrs that look like solidified drops of molten metal spattered out of the grooves during the machining process. These droplets may be of the order of $10 \mu$ in size. Several examples on one groove are shown in Lederman (1974).

The burrs will affect the perceived roughness, and this effect should be predicted by the candidate parameters if they are correct. Obviously, the effect will be proportionately stronger when the tiles are smooth than when they are rough. In other words, the most important effects of the burrs should be found for narrow-groove tiles. Without considering the candidate parameters directly, intuition dictates that a component of roughness due to the burrs, might swamp the component due to the grooves if the grooves are narrow; if the grooves are wide, the burr component will be unimportant. Hence we might expect the leveling effect for small grooves. The effect of burring on the roughness as land width is varied cannot be judged so intuitively. It depends on the model used to predict roughness. As land width increases, there are fewer grooves and fewer burred edges. The candidate parameters react differently to this fact, and must be considered separately.

The effect of burrs on the parameter D does not improve its predictive value. Consider the configuration of the skin over the groove, the burred edges, and the land, as shown in Figure 9. Particularly with the $S$-plates, there are effectively two grooves, one "official," and one "unofficial" between the burrs, where the land is supposed to be flat. The depth of finger penetration into the unofficial groove must be considered in calculating $D$. If it is greater than the penetration into the official groove, then it controls D. This will happen when the official groove is narrow compared to the land, provided that the skin does not "bottom out" on the land surface. If the skin does bottom out, then increasing finger force will not change the depth of penetration into the unofficial groove, but it will affect the depth in the official groove where bottoming out is not possible.

\section{Finger}

Figure 9. The shape taken by the idealized finger pressing on a tile with burred groove edges. Note that the skin may touch the land surface in the shallow groove between the burrs where the ideal tile has a flat land. This limitation on deformation may account for the reduced effect of finger force when the grooves are narrow.

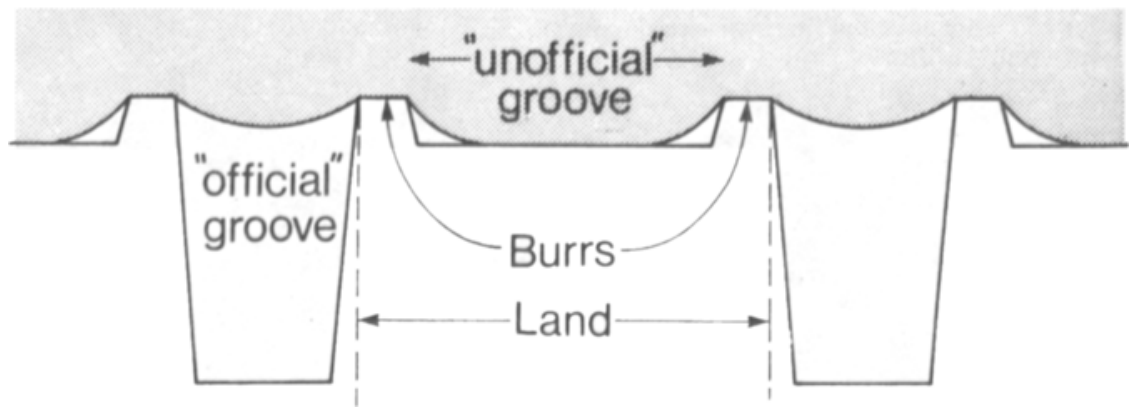


The problem with the predictions from the parameter $D$ is largely that the predicted roughness increases with increasing land width, whereas the perceived roughness decreases. The addition of the burrs does not improve this prediction. They cannot reduce the maximum depth of finger penetration into the official groove, whether the land is wide or narrow. When the land is narrow, the depth in the unofficial grooves is less likely to exceed the depth in the official grooves than if the land is wide. Hence the burrs are more likely to increase the predicted value of $D$ for wide lands than for narrow lands, making the prediction of roughness worse, not better. This further reduces the credibility of $D$ as a viable candidate, and we can now say with considerable assurance that roughness is not determined by the depth of finger penetration into the grooves.

The situation for $A_{\mathfrak{c}}$, the cross-sectional area referred to the highest point on the skin, is different. Burring improves the prediction from $A_{c}$. The calculated value of $A_{c}$ consists of two separable components, one due to the penetration of the skin into the official groove, and one due to the penetration into the unofficial groove. The predictions in Figure 7 refer only to penetration into the official groove. When these values are small and penetration into the unofficial groove is relatively large, then the unofficial components may dominate. Qualitatively, this happens when the lands are wide compared to the grooves. Apart from the leveling effect, the main difficulty with the predictions from $A_{c}$ is that its value declines too much with increasing land width. The burrs reduce this tendency, and therefore improve the prediction. It is not possible to make a quantitative assessment of the degree of improvement, especially with the EDM plates, whose burrs were not in the form of "railway tracks." However, it does seem likely that the unofficial component of $A_{c}$ could not be larger than the official component unless the lands were appreciably wider than the official groove, since the burrs themselves take up a finite width of the land. If such is the case, then the leveling effect would be predicted for $A_{c}$ only where the grooves were narrower than $0.01 \mathrm{in} .(=.25 \mathrm{~mm})$, the width of the lands in Experiment IV (Lederman, 1974). The lack of force effect would be predicted when both the unofficial grooves dominated and bottoming in these grooves occurred. Burrs do not seem very much to affect the credibility of $A_{c}$ as a candidate parameter. On the whole, $A_{c}$ seems unlikely to be the determiner of tactual roughness.

It is harder to predict exactly what effect the burring will have an $\mathrm{A}$, the cross-sectional area referred to the mean level of the skin, since the mean level varies with the depth in both official and unofficial grooves. To a first approximation, however, we can consider the contribution of the burrs as if it added linearly to the roughness caused by the official grooves. When the predicted roughness is small, the burr contribution may well dominate, but without knowing the size distribution of the burrs, this supposition cannot be checked numerically. It is clear, however, that the burr contribution will be small compared to the groove contribution when the grooves are wide. If the burr contribution dominates, the finger force should not have an important effect, because the finger probably touches the land over much of the distance between the burrs; but if the groove contribution dominates, then finger force will have its full effect. This pattern is qualitatively in correspondence with the observations, but we recognize that the explanation is not fully satisfactory, since it relies more on intuition than on calculation. All the same, A remains a plausible candidate for the stimulus for roughness. A is conceptually more readily generalized to irregular surfaces, and may perhaps be preferred for that reason.

\section{Hand Speed}

The effect of hand speed on perceived roughness is small, and consists largely of making smooth things feel slightly smoother (Katz, 1925; Lederman, 1974). If the momentary configuration of the skin dictates the percept of roughness, as the static model dictates, then this is the effect that hand speed should have. Unless there are specific mechanical resonances interacting with the vibratory stimulation involved in the deformation, the skin should deform less as it moves faster. Viscosity and inertia effects come more into play as velocities and accelerations increase. The skin has less time to conform to the idealized circular arc as the hand moves faster in comparison to the speed of surface waves on the finger. Hence the static model qualitatively predicts the effect of hand motion.

As matters stand, we cannot be more definite than to say that a model based on the momentary configuration of the skin and not on skin dynamics seems to account for the known facts of roughness perception. It is not certain yet what aspect of the deformation is the stimulus for roughness, but the most credible and, a priori, the most reasonable of those we have tested is the total amount by which the skin deviates from its resting level, and not the simple depth of deformation. We have referred in the analysis to the "cross-sectional area" of this deviation, since the test material varied only across and not along the grooves. In actuality, of course, the volume of the deviation is the parameter of interest, and would be the only calculable parameter for randomly textured surfaces. 


\section{PART II: AN EXPERIMENT WITH FRICTION}

One test of a model is whether it can produce correct but counterintuitive predictions. Many people believe that roughness depends on the coefficient of friction between the skin and the surface being judged. As we have found by querying our colleagues and acquaintances, they tend to think that the surface will seem rougher as the coefficient of friction increases. Indeed, Ekman, Hosman, and Lindstrom (1965) seemed to demonstrate that this proposition was correct. The static model, however, is insensitive to changes in the friction coefficient over quite wide ranges, regardless of the parameter used. A large increase in the friction coefficient in fact tends to cause a negligibly small decrease in the predicted values of the viable candidate parameters. This prediction implies that perceived roughness should be essentially independent of the friction coefficient, or even that it might decrease slightly as friction increases. The prediction is clearly both counterintuitive and in apparent contradiction with published data. Before the model can be allowed too much credence, then, the effect of friction must be determined in the situation for which calculations have been made.

\section{Experiment}

The roughness of grooved aluminum plates was measured by the same technique as was used in previous experiments (Lederman, 1974; Lederman \& Taylor, 1972). On this occasion, however, the plates were sometimes made slippery by the application of liquid detergent. The detergent reduced the coefficient of friction from about 0.6 to something less than 0.15 , but was found to have a negligible effect on the perceived roughness of the plates. The technique used to measure the friction coefficient is discussed in Appendix 2.

\footnotetext{
Method

Procedure and stimuli. As in the previous experiment, the tactile stimuli were placed in one end of the balance apparatus, and counterweights adjusted to give the desired touching force. In the present experiment, a touching force of $4 \mathrm{oz}(112 \mathrm{~g})$ was used thoughout. The placement of the tile was such that the touching finger (always the subject's middle finger) passed about 1 in. $(25 \mathrm{~mm})$ from the end of the tile nearest him. This permitted the experimenter to put detergent on one end of the tile without affecting the other end, so that the same tile could be used in both "soaped" ("low" friction condition) and "dry" ("high" friction condition) touching conditions. When the touching was to be "soaped," the experimenter placed with a pencil eraser a large drop of detergent on the smooth area at the side of the tile about 1 in. $(25 \mathrm{~mm})$ from the end nearest the subject. The subject's finger was placed on this drop to start the touching process. Before every trial, whether "soaped" or "dry," the subject rinsed his finger in warm water and dried it with a paper towel. Between runs, the tiles were cleaned by rinsing in warm water and then thoroughly scrubbed
}

with trichloroethylene followed by alcohol.

Two sets of electric discharge machined (EDM) plates (see Lederman, 1974) were used. One set varied in groove width by increments of $0.005 \mathrm{in} .(0.13 \mathrm{~mm})$ from .015 to $.040 \mathrm{in} .(0.38$ to $1.0 \mathrm{~mm})$ and had a constant land width of 0.01 in. $(0.25 \mathrm{~mm})$. These tiles were labeled G3 to G8 in order of increasing groove width. The other set had a constant groove width of 0.01 in. $(0.25 \mathrm{~mm})$ and a land width that varied by increments of $0.005 \mathrm{in}$. $(0.13 \mathrm{~mm})$ from 0.01 to $0.040 \mathrm{in}$. $(1 \mathrm{~mm})$. These tiles were known as L2 to L.8 in order of increasing land width. Tile L1 had a land width of .007 in. $(0.18 \mathrm{~mm})$.

The magnitude estimation procedure was the same as was used previously (Lederman, 1974; Lederman \& Taylor, 1972). At the beginning of each session, the subject was allowed to feel Plates G8 and L8, each under soaped and dry conditions. The plates were described as being "a couple of the roughest" and "a couple of the smoothest" tiles to be presented. The subject assigned numbers he felt to be appropriate for the roughnesses of these tiles and related all the roughnesses in the experiment to these assignments. Subjects were instructed to maintain the same number scale from day to day.

Experimental design. Because we wished to test both land effects and groove effects in the same sessions, the experimental design had to be rather complex. All of the land-varying tiles should, from previous results, have had roughnesses very close to one another, whereas the groove-varying set should have had quite disparate roughnesses. Clustering of stimulus magnitude may possibly cause changes in the magnitude estimation scale, in the sense that the scale is expanded where the stimuli are clustered. This seems to happen at least in category scaling (e.g., Pollack, 1964; Stevens, 1957), and could conceivably occur in the magnitude estimation of roughness, for which good experimental data seems to be lacking. The experimental design was developed to avoid such possible clustering effects, by making it always appear to the subject that he was dealing with a simple groove-varying set of eight tiles, as in the previous studies.

There were 14 different tiles in the experiment, labeled L1-L8 and G3-G8. Eight tiles were used in any run, all six of the $G$ tiles and two of the $\mathrm{L}$ tiles. The subjects were given two runs within a "period," and two periods in each session. Four sessions were run on consecutive or nearly consecutive days. Within a run, each of the eight tiles in a group was used, half "soaped" and half "dry." The soaped tiles were one of the $L$ tiles and either G3, G5, and G7, or G4, G6, and G8. In the second run of the period, the same tiles were used, but the soaped and nonsoaped conditions for each plate were reversed. For the second period of the session, two other $L$ tiles were grouped with the same six $G$ tiles. A period thus constituted on replication of the experiment for the $G$ set, but four periods were needed for a single replication of the $L$ set.

In the analysis, the data were treated as if they had come from two separate experiments, one on the $G$ set and one on the $L$ set. The only difference between the analyses depended on the difference in the number of replications. Both were full factorial designs.

\section{Results}

The combined results are shown in Figure 10. Roughness as usual increases with increasing groove width and decreases slightly with decreasing land width. The land width decline is smaller but more regular than the data from Lederman (1974; Experiment III) which were used to test the model in Part I of this paper and also seems to fit the predictions from the parameters $A_{c}$ and $A$ better than do the earlier data.

It is clear from Figure 10 that reducing the friction coefficient drastically does not result in an equally drastic reduction in the roughness of the tiles. The 
standard error of measurement in Figure 10 for a $G$ tile is about $0.01 \log$ units $(0.1 \mathrm{~dB})$, and for an $L$ tile is about $0.025 \log$ units $(0.25 \mathrm{~dB})$. If there really is a true decrease in roughness with the decreased friction, with a probability greater than 0.99 , it amounts to less than $0.12 \mathrm{~dB}$ for the $\mathrm{G}$ set and $0.75 \mathrm{~dB}$ for the $\mathrm{L}$ set. The data suggest overall that a slight increase is more likely than any decrease.

\section{Discussion}

Ekman et al. (1965) found that a 3-dB increase in the coefficient of friction resulted in a 3- to $9-\mathrm{dB}$ increase in the perceived roughness, depending on the subject. Two of his 10 subjects showed an increase as small as $1 \frac{1}{2} \mathrm{~dB}$. In the experiment reported above, we changed the coefficient of friction by at least $6 \mathrm{~dB}$ and possibly much more. Extrapolating from Ekman et al., we would have expected a decrease in perceived roughness in the neighborhood of $12 \mathrm{~dB}$, but from our data we cannot believe that a real decrease of even as much as $1 \mathrm{~dB}$ occurred. There is clearly a conflict between our results and those of Ekman et al.

In consideration of the conflict, we must discuss the nature of friction and its measurement. Friction is said to occur when one surface resists sliding over another. Its precise measurement is impossible since the term is not well defined (Feynman, Leighton, \& Sands, 1965). However, the common definition of the coefficient of friction is the ratio between the lateral force along the interface and the force normal to the interface under conditions where slip is just impending (static coefficient) or stably maintained (dynamic coefficient). This definition is adequate for our purposes, and we will ignore the distinction between static and dynamic coefficients, since the following argument applies to both.

Why does one surface fail to slide against another? There may be two separate types of answer to this question, one dealing with microscopic phenomena intrinsic to the materials of the surfaces, the other dealing with macroscopic effects related to their geometries. We deal with the macroscopic effects first. Two ridged surfaces in which the ridges of one fit into the valleys of the other will not slip easily, since the whole objects must be physically moved apart in order that the opposed ridges may pass over one another. Failing that, the ridges of at least one surface must deform bodily. Geometric distortion or movement opposed to the applied normal force must occur if parts of one object fall into hollows of the other object. To some extent, the same effects may occur at the microscopic level, but an important extra source of binding is likely to be direct molecular bonding. Atoms or molecules of one material may become bound to the surface of the other material, and the lateral force must be enough to shear these microscopic welds before the surfaces can move against one another. At a microscopic level, the

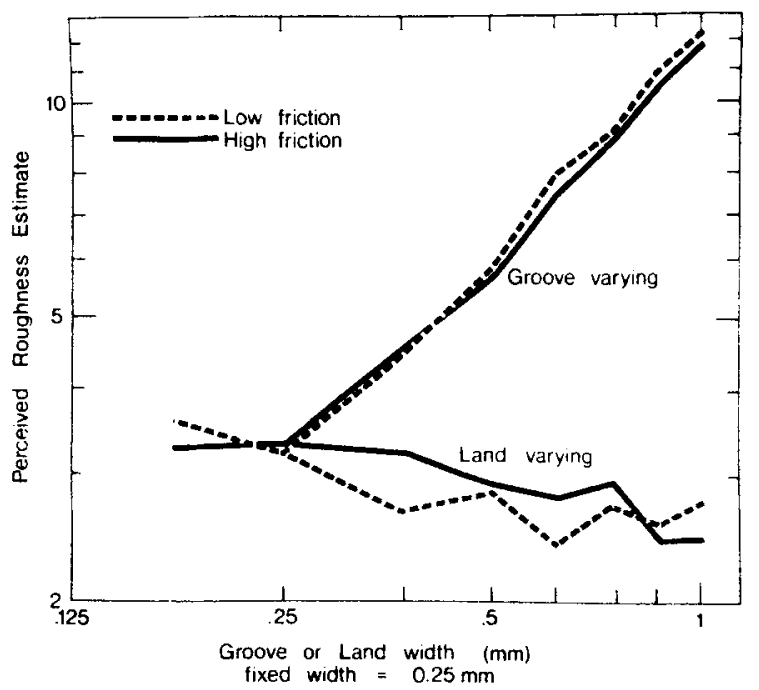

Figure 10. Perceived roughness as a function of groove and land width for low-friction and high-friction conditions.

surfaces of particular materials may have an intrinsic roughness below which it is impossible to smooth them. This intrinsic surface roughness may be due to the crystalline structure of the material, and materials made of large crystals may be smooth even to an atomic level. Microcrystalline materials, however, can usually not be so smoothed by ordinary means. Both molecular binding and microscopic roughness intrinsic to the materials may determine lower bounds to how easily two materials slide against one another. We may thus distinguish between microscopic friction, that is almost intrinsic to the materials sliding over one another, and macroscopic friction, which depends on the geometric forms of the sliding objects.

In consideration of the discrepancy between our results and those of Ekman et al. (1965), the distinction between microscopic (material) friction and macroscopic (geometric) friction looms large. Both our model and our measurements consider only microscopic friction, the friction between the finger skin and the supposedly smooth surface of the lands on the tiles. Ekman et al. do not describe their technique for measuring friction, but they used among their stimulus materials sand papers of various grades. These sandpapers were probably made of essentially the same materials no matter what their grade, and hence should have had the same microscopic coefficients of friction. All the variations they measured must have been in the macroscopic coefficient. In our experiment, this would have been equivalent to determining the friction coefficient by measuring the resistance of the finger to movement across, rather than along the grooves. The finger would have been impeded by the very distortion we believe causes the percept of roughness. We would 
have measured macrofriction and perhaps have predicted the perceived roughness. A proper control experiment to test by the methods of Ekman et al. whether the microscopic coefficient of friction is implicated in the perception of roughness would be to make replicas of his stimuli in various materials. Provided that the materials were equally rigid, they should all have about the same perceived roughness as the original from which they were copied.

\section{CONCLUSION}

\section{General Discussion}

We have proposed a model which accounts for most of the available data on the perception of roughness. The model was used to predict correctly that the coefficient of friction between skin and surface should not affect perceived roughness very much. But successful prediction does not necessarily imply the correctness of the model. It would be helpful if we could observe directly the configuration of the skin as it passes over the grooves, but we have so far been unable to achieve this end. It is quite possible, for example, that roughness does depend on dynamic effects, but that these effects correlate with the static deformation that would be achieved if the finger rested on the tile for a long time. There is considerable evidence, for example, that the sensitivity to punctate vibration increases with increasing frequency to some $250 \mathrm{~Hz}$ (e.g., Goff, 1967; Verrillo, 1962), and it is conceivable that this increased sensitivity might just offset a decrease in the real deformation of the skin at higher and higher speeds of movement. The fundamental frequencies involved in Lederman's hand-speed study (1974, Experiment II) range from $10 \mathrm{~Hz}$ to around $700 \mathrm{~Hz}$, and at the highest frequencies there is a suggestion that things seem smoother than expected. This effect could be consistent with either a static or a dynamic model.

Having entered this caveat, we note that the static model is reasonably successful, and if a dynamic model recreates its predictions, no choice can be made between them. New conditions must be found in which the predictions differ. Such conditions could be achieved if it proved possible to construct a surface which should be everywhere in contact with the skin. According to the static model, finger force should not affect the perceived roughness of such a surface, and neither should hand speed. However, if a dynamic model is more nearly correct, then the skin would not necessarily touch the surface everywhere, particularly at higher hand speeds. Roughness should increase with hand speed as long as the skin remains in contact with the surface, and should increase with finger force only for higher speeds, when the skin is not always in contact. Production of such stimuli is not easy.

\section{Summary}

Analytic consideration of the form taken by an idealized finger pressed onto a grooved surface suggested 11 possible parameters as the stimulus for roughness. Data from previously published studies indicated that for wide enough grooves, roughness was approximately a function of $\mathrm{FG}^{4}$ where $\mathrm{F}$ is the applied finger force and $G$, the groove width. An approximate analysis permitted 7 of the candidate parameters to be discarded, and the other 4 were analyzed more closely. By comparison with the experimental data, the most likely candidates were the volume of the deviation of the skin from its mean resting level, and the volume of the deviation measured from the highest level of the skin. The candidates were all fairly insensitive to the coefficient of friction between skin and surface, in contradiction with intuition and with published data on the effect of friction on perceived roughness. An experiment demonstrated nearly zero effect of the coefficient of friction on perceived roughness, in agreement with the predictions of the model. The discrepancies with prior data were considered in terms of the difference between friction due simply to the slipping materials and friction due to the geometric form of the slipping objects. In conclusion, it was suggested that a test between the static model and possible competing dynamic models might be possible.

\section{APPENDIX 1: METHOD OF CALCULATING A AND D}

Details of the method of calculating A, the total cross-sectional area of skin deformed from the resting position of the finger may be found in Lederman (1973). Here we present only the gross steps involved. In the process, we also calculate the parameter $D$.

Referring to Figure 1 for the symbols, it is possible to show geometrically:

noting that

$$
\mathrm{D}=\mathbf{r}-\mathbf{H}
$$

(where $\mathrm{H}$ is the vertical height from the center of the arc to the level of the land),

$$
\mathrm{r}=\mathrm{G} / 2 \sin \Theta
$$

and

$$
\mathbf{H}=\mathbf{r} \cos \Theta
$$

then

$$
\begin{aligned}
\mathrm{D} & =\mathbf{r}(1-\cos \Theta) \\
& =\mathrm{G}(1-\cos \Theta) / 2 \sin \Theta
\end{aligned}
$$

The area of the segment $A_{c g}$, which we now denote as $B$ for convenience, is given by 


$$
B=r^{2}(\Theta-1 / 2 \sin 2 \theta)
$$

It is possible to show that the mean resting skin position is given by the depth $\mathrm{d}$ below the land surface, where

$$
\mathrm{d}=\mathrm{B} /(\mathrm{L}+\mathrm{G}) \text {. }
$$

Now the width of the segment $A_{g}$ is given by $x$, where $x=D-d$, or

$$
x=[G(1-\cos \theta) / 2 \sin \theta]-\left[r^{2}(\Theta-1 / 2 \sin 2 \theta) /(L+G)\right] .
$$

Using the angle subtended at the arc center by the segment $A_{g}$, it is then possible to show that

$$
A_{g}=1 / 2 r^{2}\left[\sin ^{-1}\left(2 p-p^{2}\right)^{1 / 2}-(1-p)\left(2 p-p^{2}\right)^{1 / 2}\right]
$$

where $p=x / r=(1-\cos \Theta)-\{[(\Theta / 2 \sin \Theta)-\cos \Theta] /[1+(L+G)]\}$. $A$ is then given by $\left(W A_{g}\right)(G+L)$, where $W$ is the equivalent width of the finger pad, as determined from the pad area measure. At this point, we have found $A$ as a function of $\Theta, G$, and $L$. $G$ and $L$ are known, but $\Theta$ must be determined for any particular force condition.

It is possible to determine $\mathrm{kfg}_{\mathrm{g}}$ in terms of $\Theta$. The full derivation is rather complex Lederman, 1973), and it suffices to note here that the final expression is

$$
\mathrm{kf}_{\mathbf{g}}=\frac{2 \mathrm{G}^{2}(\theta-\sin \theta)}{\mathrm{G}^{2}+\mathrm{LG}-1 / 2 \mathrm{~L}^{2} \mu \sin \theta}
$$

where $\mu$ is the coefficient of friction between the skin and the land surface. Expression 4 is not easy to solve for $\Theta$ in terms of $k f_{g}$, but numeric values of $\mathbf{k f}_{\mathrm{g}}$ may readily be calculated for any value of $\Theta$, on the assumption of a particular value of $\mu$. Hence, to determine the value of $\Theta$ appropriate to an experimental value of $\mathrm{kf}_{\mathrm{g}}$, an iterative computation was used to obtain successive approximations to the desired $\mathbf{k f}_{\mathrm{g}}$. Once the value of $\Theta$ had been determined which gave the correct value of $\mathbf{k f}_{\mathrm{g}}$, it was substituted into the expression for $A$, giving the value of $\$$ for that $\mathbf{k f}_{\mathrm{g}}$. The values for the other candidates were determined similarly, although their formulas were somewhat simpler (Lederman, 1973). For example, D is obtained directly by substituting the appropriate value of $\Theta$ in Equation 1.

\section{APPENDIX 2: \\ THE MEASUREMENT OF FRICTION BETWEEN TILE AND SKIN}

The coefficient of friction between two surfaces is the ratio between a force along the interface and a force across (perpendicular to) the interface. The forces may be determined when the surfaces are in smooth relative motion, in which case the coefficient is called the dynamic coefficient of friction, or they may be measured in the condition where the lateral force is the largest force that just does not cause the objects to slide against one another, in which case the coefficient is called the static coefficient of friction.

It is relatively easy to measure the friction coefficient between two inanimate objects. One is laid on the other and pushed sideways. The weight of the upper object is the normal force and the push is the lateral force. Their ratio is the coefficient. When one of the objects is animate, such as a finger, the problem is trickier, since the normal force is determined not by the weight of the finger but by the balance of muscular forces applied to it. Also, the lateral force is applied in part by the muscular balance. Hence, one cannot readily measure the normal and lateral force components.

Our method of measuring the coefficient required the construction of an apparatus which permitted the measurement of the normal and the lateral forces. A cross-section of the apparatus is seen in Figure A-1. A tile is held vertically between the finger and two wheels against which it could run freely. The force applied to the wheels was measured by a force gauge. This force was constrained to be normal to the tile, because any lateral component (in the vertical direction) would have caused the wheels to rotate and the tile to move. If the finger did not apply sufficient force, the weight of the tile would cause it to slip down. The lateral force supplied by finger-tile friction balanced the weight of the tile if the finger force was large enough. This balance was demonstrated by the fact that the tile did not move, since the resultant force on the wheel was necessarily applied normal to the wheel circumference.

To measure the friction coefficient, the subject pushed the tile with the grooves aligned vertically hard against the wheel while the experimenter watched the force gauge. As the subject gradually reduced his applied force, the tile would slip and the experimenter would not the force at which this happened. Knowing the weight of

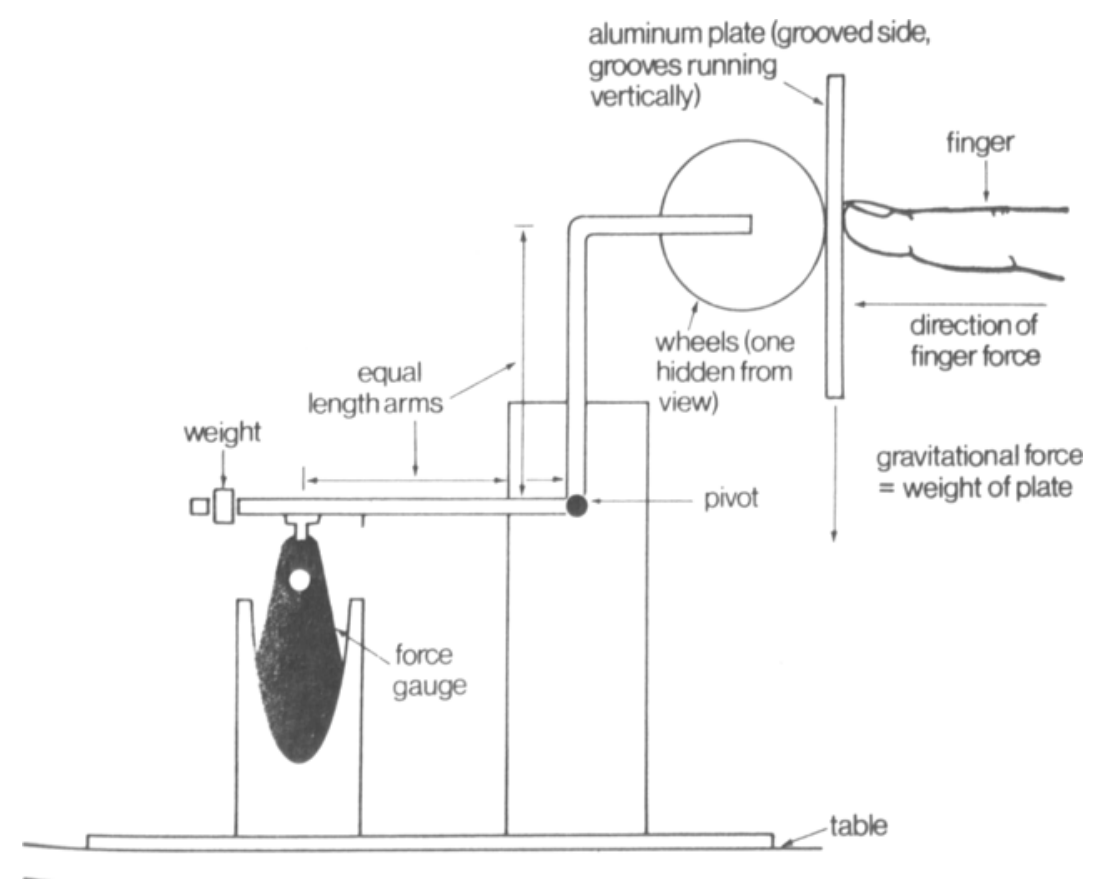

Figure A-1. The apparatus used to measure the coefficient of friction between finger and tile. For description of its use, see Appendix 2. 
the tile, the coefficient of static friction could then be calculated. With various subjects, the coefficient was usually close to 0.6 . When the fingers were soaped, reproducing the touching conditions in the friction experiment, the applied force when the tile slipped was usually too large to measure with the force gauge available, and we can only say that the coefficient was less than about 0.15 - probably much less.

\section{REFERENCES}

Ekman, G., Hosman, J., \& Lindstrom, B. Roughness, smoothness and preference: A study of quantitative relations in individual subjects. Journal of Experimental Psychology, $1965,70,18-26$.

Feynman, R. P., Leighton, R. B., \& Sands, M. The Feynman lectures on physics. Reading, Mass: Addison-Wesley, 1965.

GoFF, G. D. Differential discrimination of frequency of cutaneous mechanical vibration. Journal of Experimental Psychology, 1967, 74, 294-299.

Katz, D. Der Aufbau der Tastwelt (The world of touch). Zeitschritt für Psychologie, 1925, 11, I-XII and 1-270.

LEDERMAN, S. J. The perception of surface roughness by touch. Unpublished PhD dissertation, University of Toronto, 1973.

Lederman, S. J. Tactile roughness of grooved surfaces: The touching process and effects of macro- and microsurface structure. Perception \& Psychophysics, 1974. 16. $385-395$.
Lederman, S. J., \& Taylor, M. M. Fingertip force, surface geometry, and the perception of roughness by active touch. Perception \& Psychophysics, 1972, 12, 401-408.

Moore, T. J., \& Mundie, J. R. Measurement of specific mechanical impedance of the skin: Effects of static force, site of stimulation, area of probe, and presence of a surround. Joumal of the Acoustical Society of America, 1972, 52, 577-584.

Pollack, I. Neutralization of stimulus bias in auditory rating scales. Journal of the Acoustical Society of America, 1964, 36, 1272-1276.

Stevens, S. S. On the psychophysicsl law. Psychological Review, 1957, 64, 153-181.

Taylor, M. M., Lederman, S. J., \& Gibson, R. H. Tactual perception of texture. In E. C. Carterette and M. P. Friedman (Eds.), Handbook of perception (Vol. 3). New York: Academic Press, 1973.

TREgeAR, R. T. The physical functions of the skin. New York: Academic Press, 1966.

VERrillo, R. T. Effect of contactor area on the vibrotactile threshold. Journal of the Acoustical Society of America, 1963. 35, 1962-1966.

Von Gierke, H. E., Oestreicher, H. L., Franke, E. K., Parrack, H. O., \& von WitTERN, W. W. Physics of vibrations in livi.ng tissues. Journal of Applied Physiology, $1952,4,886-900$.

Yamada, H. In F. G. Evans (Ed.), Strength of biological materials. Baltinore: Williams, 1970.

(Received for publication March 6, 1974; revision received May 7, 1974.) 